\title{
Construcción y validación de una escala de buenas prácticas interculturales en Trabajo Social
}

\author{
Construction and Validation of a Scale for Assessing Best \\ Intercultural Practices in Social Work \\ Octavio Vázquez-Aguado \\ Universidad de Huelva \\ octavio@uhu.es
}
Manuela A. Fernández-Borrero
Universidad de Huelva manufb13@gmail.com
Miriam Fernández-Santiago
Universidad de Granada mirfer@ugr.es

\author{
Patricia Vaz-García \\ Universidad de Huelva \\ patri.vaz@gmail.com
}

Recibido: 01/10/2010

Revisado: 05/07/2011

Aceptado: 28/11//2011

Disponible on line: 15/02/2012

\begin{abstract}
Resumen
El objetivo principal de este artículo es mostrar el proceso de construcción y validación de un instrumento de medida que permite delimitar qué puede ser considerado «buena práctica» en relación a proyectos interculturales. Este trabajo se enmarca dentro de una investigación más amplia denominada «Mapa de buenas prácticas interculturales en Andalucía» y que fue financiada por la Dirección General de Coordinación de Políticas Migratorias de la Consejería de Gobernación de la Junta de Andalucía. La escala validada está formada por 32 ítems distribuidos en cuatro dimensiones: teórica (con tres subdimensiones: factor contextual, factor concepción de la diversidad y factor de elementos de diversidad), ético-ideológica, socio-política y una última de empatía intercultural. Se incluyen también otras cuestiones relacionadas con datos de identificación de los proyectos participantes. Esta escala ha sido pilotada en proyectos de intervención social de diferentes sectores de actuación que comparten la visión intercultural (empleo, educación, salud, etc.) y que se desarrollan en Andalucía (España). Con los datos obtenidos hemos alcanzado evidencias de validez de constructo y elevada fiabilidad del instrumento diseñado.
\end{abstract}

Palabras claves: acción intercultural, buena práctica, evaluación de proyectos, validación de escalas, intervención social.

\begin{abstract}
This article reports on the construction and validation process of an instrument that enables the placement of intercultural projects on a scale of Best Practices. The validated scales includes 32 variables divided into four categories: theoretical (subdivided further into three categories: contextual, concept of diversity and elements of diversity); ethicalideological, sociopolitical and intercultural empathy. Questions related to project identification are also included. The scale was piloted in social intervention projects in different sectors sharing the intercultural vision (employment, education, health, etc.) and was developed in Andalusia (Spain). The data obtained show evidence of construct validity and high reliability of the instrument as designed.
\end{abstract}

Key words: Intercultural action, Best practice, Project assessment, Scale validation, Social intervention.

Referencia normalizada: Vázquez-Aguado, O., Fernández-Borrero, M. A., Fernández-Santiago, M., Vaz-García, P. (2012): «Construcción y validación de una escala de buenas prácticas interculturales en Trabajo Social». Cuadernos de Trabajo Social, 25(1): 159-171.

Sumario: Introducción. 1. Método. 2. Resultados. 3. Discusión. 4. Referencias bibliográficas. 


\section{Introducción}

La tesis de que la dimensión cultural de los problemas sociales debe tenerse en cuenta en el diseño e implementación de Servicios Sociales viene siendo defendida con fuerza en las últimas décadas (Bernal y Sáez-Santiago, 2006; Tricket, Watts y Birman, 1994; Martínez, Martínez y Calzado, 2006). Su principal sustento está en el hecho de que si la conducta humana es aprendida y desplegada en un contexto cultural, toda intervención que quiera ser efectiva ha de prestar atención al entorno cultural donde están inmersos los individuos (Perdersen, 2003). De este modo, articular lo intercultural con la intervención supone fortalecer el vínculo que se establece entre una estructura social y la consolidación de las aspiraciones del reconocimiento de la diversidad cultural (Martuccelli, 2004).

La prestación de servicios culturalmente apropiados es una preocupación que ha ido creciendo en la medida que poblaciones de origen extranjero se han asentado en países distintos al de origen, lo que ha motivado un interés por desarrollar servicios culturalmente sensibles, así como la puesta en marcha de estrategias de intervención que contemplen la diversidad cultural y prácticas de asesoramiento y gestión intercultural (Pratt y Apple, 2007). Pero, para ello, la constatación de la diversidad sobre un territorio es condición imprescindible. Tomar conciencia de esta pluralidad, de los problemas y beneficios que aporta, es el punto de partida para plantearnos la naturaleza del proceso a seguir en la intervención social, que se ve también condicionada por los agentes y los objetivos finales a alcanzar con la acción intercultural (Vázquez, 2001).

En este sentido, el punto final de toda acción intercultural es conseguir que su plano fáctico se aproxime a la conceptualización teórica de la interculturalidad en el desarrollo de relaciones de individuos de diversas culturas en un mismo espacio y en los diferentes ámbitos de la estructura social. La interculturalidad debe entenderse pues, como el objetivo o fin último, mientras que la acción intercultural es el medio con el que se puede alcanzar dicho objetivo (Vázquez, Fernández, Fernández, Vaz y León, 2008). La acción intercultural la entendemos como una práctica social en la que intervienen individuos con referentes culturales diversos y que pretende la comprensión positi- va de esta diversidad cultural persiguiendo la cohesión social, sin obligar por ello a la aceptación y valoración de todas y cada una de las prácticas culturales que existen en el territorio. Como afirma Panikkar (2006), la interculturalidad se basa en la situación dinámica del hombre que, consciente de la existencia de otras personas, valores y culturas, sabe que no puede aislarse en sí mismo.

En cuanto a la planificación de las instituciones en clave intercultural, en la bibliografía existente se suelen citar los siguientes elementos como fundamentales: el apoyo de la institución en la que se presta el servicio; la actualización de la misión y las políticas de la institución; la mejora del conocimiento que el personal de la institución tiene acerca de las poblaciones diversas que se localizan en la comunidad; la evaluación y la preocupación por mejorar las habilidades interculturales del personal que intervienen en el proyecto; la diversidad cultural del personal que presta el servicio; el conocimiento previo por parte de los profesionales sobre las necesidades y creencias de los clientes con los que se va a trabajar; la adaptación de los procedimientos, la infraestructura y entorno físico; la facilitación de la comunicación verbal y escrita o la colaboración con los líderes de las comunidades diversas que existen en el territorio. Finalmente, no debe olvidarse la evaluación de los resultados por parte de las instituciones, la clara presentación de sus objetivos y la difusión de recomendaciones que ayuden a mejorar las prácticas interculturales de las organizaciones (U.S. Department of Health and Human Service, 2001; Julve y Palomo, 2005; Whealin, 2008.)

En este trabajo defendemos que una Buena Práctica Intercultural (BPI) incorporará, por una parte, las características de una buena práctica (entre otras, la representación de los sujetos implicados en la acción, la viabilidad real del desarrollo de la acción así como que sea accesible, la sostenibilidad temporal y económica y la consecución de cohesión social), así como las exigencias específicas relativas a las relaciones interculturales. Esta exigencias hacen referencia a la existencia de un marco de valores y una declaración de principios que forma parte de la planificación del proyecto/actividad y que deberá contar para su elaboración con todos los agentes implicados en su buen desarrollo (Barret, 2003). 
También contemplan la gestión de la diversidad cultural por parte de los agentes en contacto, a partir de unos criterios básicos de: justicia social, igualdad de oportunidades, libertad individual, cohesión social y valores democráticos.

Estas buenas prácticas se enmarcan en contextos en los que la tensión social puede derivarse de la falta de conciencia o rechazo a lo intercultural. Es en estos espacios en donde surgen entidades colectivas que, cargadas de buenas intenciones, carecen de un marco de referencia para estructurar su labor favorecedora de la convivencia intercultural. Dichas entidades se limitan a observar carencias en su entorno inmediato $\mathrm{y}$ a intervenir con soluciones que, en la mayoría de los casos, pierden en efectividad a causa de la carencia de un enfoque integral.

Las intervenciones en sí, por otro lado, deben ser evaluadas dentro de un marco tanto ideológico como legal que requieren de un segundo instrumento de evaluación institucional. El aspecto legal marcaría el mínimo exigido para la consideración de buena práctica intercultural, incluyendo aspectos como la no discriminación por indicadores culturales que se recogen en la constitución española y legislación vigente (religión, raza, ideología, género, etc.). El aspecto ideológico marcaría la optimización de la buena práctica intercultural, e incluiría aspectos aún no recogidos en la legislación vigente, como: la discriminación positiva por motivos interculturales tales como la lengua, la nacionalidad de origen, la ciudadanía, etc).

El diseño de dicho instrumento debe tener tres marcos de referencia: el de la legalidad vigente, el de la definición de buena práctica intercultural, y el de instrumentos similares previamente desarrollados para áreas de intervención social específicas (dentro del área de la interculturalidad, el aspecto educativo es el más desarrollado, puesto que se hace imperativo en la enseñanza de idiomas extranjeros).

Considerando que es necesario evaluar las acciones e iniciativas que se ponen en marcha en el marco de este proyecto, se ha elaborado una escala que permite medir las buenas prácticas interculturales, definiendo cuáles son las dimensiones básicas y los aspectos fundamentales que estas prácticas deben reunir. Al tratarse de un proceso de construcción y validación se contempla también como objetivo, tal y como expone Elosua (2003), recoger las sufi- cientes evidencias que puedan prestar una base científica a la interpretación de las puntuaciones en un uso concreto.

A continuación mostramos el proceso de construcción y validación de la escala, analizando la dimensionalidad del instrumento diseñado para determinar el mínimo número de estructuras necesario para explicar la máxima varianza observada. Se trataría de definir un modelo linealmente independiente que identificara en este caso una buena práctica intercultural, basado en la reproducción de las matrices de covarianzas o correlaciones entre las variables incluidas (Elosua, 2003).

\section{Método}

\subsection{Participantes}

Los participantes del presente trabajo son proyectos interculturales desarrollados por diferentes actores sociales. Para la definición del universo de proyectos a evaluar los investigadores consensuaron una serie de criterios para su localización y posterior construcción de la base de datos. Estos criterios fueron: proyectos iniciados en 2005 o posteriormente, con un año de funcionamiento al menos, con criterios interculturales básicos (diferencias culturales en sus usuarios, objetivos, etc.), desarrollados en la comunidad autónoma de Andalucía y objeto de algún tipo de financiación pública de parte de algunas de las administraciones competentes en la materia. Con estos criterios se localizaron proyectos en toda la geografía andaluza, construyéndose con ellos la base de datos inicial y seleccionando aleatoriamente 50 de ellos, con los que se contactó mediante correo electrónico y, en su defecto, por correo ordinario.

Han participado en el proceso de pilotaje y validación, por lo tanto, 50 proyectos, contando con la colaboración de 38 de ellos, que suponen el 76 por ciento del total del pretest realizado. Hay que tener en cuenta que es un objeto de investigación de difícil acceso, dado que se están evaluando proyectos y no individuos. De los 38 proyectos que finalmente participaron, el 31,6 por ciento está relacionados con los Servicios Sociales; un 31,6 por ciento está vinculado a acciones educativas; un 28,7 por ciento a un conjunto amplio de intervenciones no clasificables en estas categorías que 
señalamos y los restantes, el 7,9 por ciento, son proyectos relacionados con el empleo. Desde el punto de vista de la titularidad legal y responsabilidad de los proyectos, en el 94,7 por ciento la ejecución la han asumido entidades sociales (asociaciones, fundaciones, ONG, etc.) y en el 5,3 por ciento las administraciones públicas (mancomunidades, diputaciones, etc.). En cuanto a la distribución geográfica, el mayor porcentaje de proyectos se localiza en las provincias de Córdoba (el 28,9 por ciento), Huelva (el 26,3 por ciento) y Almería (el 13,2 por ciento). En Jaén y Málaga hay el 7,9 por ciento de proyectos en cada una, el 5,3 por ciento se localiza en Cádiz y el 2,6 por ciento se ubica en Sevilla.

La aplicación de la escala ha sido realizada mediante correo electrónico y completada por el responsable del proyecto a evaluar y, en su defecto, por el responsable de la entidad que lo desarrolla.

\subsection{Instrumento}

La Escala de Buenas Prácticas Interculturales (EBPI),fruto del estudio que hemos realizado, tiene una estructura dimensional basada en una adaptación de los estudios sobre competencia comunicativa intercultural y sensibilidad intercultural (SI), elaborados por Milton J. Bennet (1986), que propone el análisis de la comunicación intercultural en tres niveles: 1) la comprensión y el conocimiento de la diferencia cultural; 2) la aceptación de esta diferencia; y 3) la estimación de la misma a través de empatía y la consideración de la interculturalidad como un valor positivo. La escala está basada en una adaptación de los estudios sobre competencia comunicativa intercultural y sensibilidad intercultural elaborados por Chen y Starosta (2000) y Bennet (1986), así como las dimensiones de la interculturalidad definidas por Liévano (2003): la dimensión teórica vin- culada al reconocimiento de la diversidad; la ideológica que defiende la igualdad en la diversidad; la ética que propone el fomento de la interacción; la sociopolítica que favorece la dinámica de transformación social y la educativa que plantea la interacción cultural en condiciones de igualdad.

Es posible, desde nuestra óptica, establecer una correlación entre las tres aportaciones contempladas a partir de las cinco dimensiones básicas que inicialmente componen nuestra escala (que posteriormente quedaron reducidas a cuatro en el instrumento definitivo). Los elementos cognitivos de Chen y Starosta, así como el comprensivo de Bennet se corresponden con las dimensiones teórica y ético-ideológica que propone Lievano; las aportaciones relativas a los elementos conductuales y de aceptación de la diversidad están vinculados a la dimensión sociopolítica y los aspectos afectivos y de apreciación de la diferencia se vinculan a la dimensión empática presente en la escala que elaboramos.

Considerando los modelos mencionados previamente que nos han servido de base en la construcción de la Escala de Buenas Prácticas Interculturales (EBPI), hemos definido cinco dimensiones en el instrumento construido:

a) Teórica: reconocimiento de la diversidad.

b) Ético- ideológica: defensa de la igualdad y fomento de la interacción.

c) Sociopolítica: dinámica de transformación social.

d) Empática: promoción de procesos educativos que plantean la interacción cultural en condiciones de equidad.

e) Interculturalidad como valor positivo

En la Tabla 1 se pueden observar las aportaciones de cada uno de estos modelos a la construcción de la escala.

\begin{tabular}{|c|c|c|}
\hline $\begin{array}{c}\text { Nivel } \\
(\text { Hammer/Chen \& Starosta })\end{array}$ & $\begin{array}{c}\text { Componente } \\
\text { (Chen \& Starosta) }\end{array}$ & $\begin{array}{c}\text { Dimensión } \\
\text { (Liévano) }\end{array}$ \\
\hline Cognición & Cognitivo & Teórica \\
& Ideológica \\
Ética \\
Aceptación & Conductual & Sociopolítica \\
Apreciación & Afectivo & Empática \\
\hline
\end{tabular}

Tabla 1. Aportación de cada modelo a la escala 
La escala estaba inicialmente compuesta por 50 ítems (17 de la dimensión teórica, 13 de la ético-ideológica, 8 de la sociopolítica, 5 de empatía y 7 de interculturalidad como valor positivo). El instrumento diseñado es de tipo Likert de 5 puntos, donde 1 corresponde a «totalmente en desacuerdo» y 5 a «totalmente de acuerdo». Esta escala pretende valorar la adecuación de un proyecto o iniciativa de intervención (social, cultural, académico, laboral, etc.) al concepto de buena práctica intercultural. Además incorpora una serie de cuestiones relativas a datos de identificación del proyecto.

Tras la definición inicial del instrumento, en una segunda fase, se llevó a cabo la consulta a expertos mediante el método de congruencia ítem-objetivo. Con las aportaciones realizadas, se modificó la escala inicial redefiniendo algunos ítems, incluyendo o eliminando otros.

Tras este proceso, llevamos a cabo el pilotaje y, con los resultados arrojados en el análisis posterior y que son objeto de comunicación en este trabajo, se construyó la versión definitiva de la Escala de Buenas Prácticas Interculturales (EBPI), que está compuesta por 32 ítems más los datos de identificación de los proyectos. Los 32 ítems distribuidos en las di- ferentes dimensiones se pueden contemplar en la Tabla 2.

\subsection{Análisis de datos}

Los datos fueron analizados con el programa estadístico SPSS 14.0, empleándose correlaciones, análisis de fiabilidad y análisis factoriales exploratorios (AFE).

\section{Resultados}

El primer estadio del análisis de los datos fue la recodificación de aquellos ítems que puntuaban en sentido inverso a lo interpretado en la escala, logrando así que todos mantuvieran el mismo sentido en la escala de puntuación. A continuación, como medida aproximativa, se analizaron los estadísticos descriptivos univariados de los ítems agrupados en sus dimensiones. Calculando una media ideal y desviación típica máxima, podríamos identificar aquellos ítems con medias y desviaciones típicas más alejadas de la ideal. En la mayoría de las dimensiones los ítems presentan en su conjunto medias y desviaciones típicas bastante elevadas, lo que puede ser un reflejo de aquellos aspectos fundamentales compartidos por los proyectos vinculados a la noción de interculturalidad.

\section{Dimensión teórica: Factor contextual}

1. El proyecto/actividad se desarrolla en un contexto multicultural.

2. El proyecto/actividad considera el lugar que los agentes implicados ocupan en la estructura social.

3. El proyecto/actividad incluye entre sus objetivos fomentar el reconocimiento de la diversidad cultural existente en el contexto de intervención.

4. Se considera importante la realización de un estudio previo de la diversidad cultural de los colectivos en contacto.

5. El proyecto/actividad incluye entre sus objetivos fomentar el entendimiento de las diversas culturas que coexisten en el contexto en que se ejecutan.

\section{Dimensión teórica: Factor concepción de la diversidad}

6. El proyecto/actividad considera la posible existencia de prácticas discriminatorias basadas en diferencias culturales.

7. Se realiza una previsión de posibles puntos de divergencia y conflicto entre los diferentes colectivos culturales que comparten el contexto de intervención.

8. El proyecto/actividad identifica la existencia y la naturaleza de prejuicios basados en diferencias culturales.

9. El proyecto/actividad identifica la existencia y naturaleza de prácticas discriminatorias basadas en diferencias culturales

10. El proyecto/actividad condena explícitamente estereotipos y mensajes racistas.

Tabla 2. Escala de Buenas Prácticas Interculturales. 


\section{Dimensión teórica: Factor elementos de diversidad}

11. Se consideran el nivel de formación de los actores del proyecto/actividad.

12. Se considera el nivel de formación de los beneficiarios del proyecto/actividad.

13. Se consideran las posibles diferencias entre los niveles y áreas de formación de los actores y beneficiarios del proyecto/actividad.

14. Se considera la confesión religiosa de todos agentes implicados en la planificación y ejecución del proyecto/actividad.

15. Se consideran los hábitos alimentarios de los agentes implicados en el proyecto/actividad.

16. Se consideran los hábitos higiénicos de los agentes implicados en el proyecto/actividad.

\section{Dimensión ético-ideológica}

17. El proyecto/actividad incluye entre sus objetivos la formación lingüística de las minorías culturales en el idioma usado por la comunidad de acogida.

18. El proyecto/actividad incluye entre sus objetivos proporcionar a las minorías culturales una formación en valores éticos propios de la cultura de acogida.

19. El proyecto/actividad incluye entre sus objetivos proporcionar a las minorías culturales una formación en valores cívicos propios de la cultura de acogida.

20. El proyecto/actividad incluye entre sus objetivos proporcionar a las minorías culturales una formación académica/profesional de acuerdo con el modelo metodológico de la cultura de acogida

21. El proyecto/actividad incluye entre sus objetivos fomentar la tolerancia por las diversas culturas que coexisten en el contexto en que se ejecutan.

\section{Dimensión sociopolítica}

22. El proyecto/actividad incluye entre sus objetivos prevenir posibles conflictos entre individuos con patrones de referencia cultural diferentes que coexisten en el contexto en de intervención.

23. El proyecto/actividad incluye entre sus objetivos fomentar el respeto por las diversas culturas que coexisten en el contexto de intervención.

24. El proyecto/actividad promueve actuaciones contra los mutuos prejuicios y actitudes discriminatorias desarrollados y sostenidos por las diversas culturas que coexisten en el contexto en que se ejecutan.

25. El proyecto/actividad incluye entre sus objetivos la no discriminación entre sus agentes implicados por razones de diferencia cultural.

26. El proyecto/ actividad incluye la participación/pertenencia de individuos de culturas diferentes a la propia.

27. El proyecto/actividad tiene previsto un protocolo de actuación para abordar la solución de posibles conflictos interculturales.

28. El proyecto/actividad fomenta la pluralidad cultural entre sus agentes implicados

\section{Dimensión empática: interculturalidad como valor positivo}

29. El proyecto/actividad incluye entre sus objetivos fomentar las relaciones entre las diversas identidades culturales que coexisten en el contexto en que se ejecutan.

30. El proyecto/actividad incluye entre sus objetivos la celebración de eventos culturales que implican al menos a más de una cultura de referencia.

31. El proyecto/actividad incluye entre sus objetivos la difusión de las señas de identidad culturales de grupos de individuos con diversas culturas de referencia (historia, arte, tradiciones, valores, creencias populares, etc.

32. El proyecto/actividad incluye entre sus objetivos la celebración de encuentros interculturales entre grupos de individuos con diversas culturas de referencia.

Tabla 2 (cont.). Escala de Buenas Prácticas Interculturales. 
Posteriormente, el análisis de correlaciones entre los ítems de la escala inicial por dimensiones y de las puntuaciones globales de cada dimensión, nos mostró que un total de once ítems no presentaban correlaciones significativas. Veamos cómo afectaba esta cuestión a cada dimensión:

- en el caso de la dimensión teórica, considerando las correlaciones no significativas, el contenido semántico de los ítems y la extensión de la dimensión, el equipo investigador se planteó realizar una revisión bibliográfica más profunda que posibilitó la subdivisión de esta dimensión en tres factores: Contextual, que hace referencia al contexto como marco físico y escenario de la intervención. Concepción de la diversidad, que afecta al concepto de diversidad en que se basa el proyecto y el modo de tenerlo en cuenta en la planificación y desarrollo del mismo. Elementos de diversidad, referidos a aspectos concretos generadores de diversidad.

- En la dimensión ético-ideológica existían cinco ítems que destacaban por sus bajas correlaciones. Uno de estos ítems (El proyecto/actividad considera el lugar que los agentes implicados ocupan en la estructura social) pasó a ser incluido en el factor contextual de la dimensión teórica por su contenido semántico, comprobando que correlacionaba satisfactoriamente con los ítems de este factor. La decisión de supresión o no de estos ítems tenía que tomarse cuando se realizasen los análisis factoriales.

- En la dimensión sociopolítica existían dos ítems con correlaciones no significativas con el resto de la dimensión (El proyecto/actividad facilita información a las minorías culturales exclusivamente en el idioma de la comunidad de acogida y El proyecto/actividad facilita información a los destinatarios exclusivamente en su propio idioma).

- En la dimensión sobre empatía intercultural, tan sólo un ítem no presentaba ninguna correlación significativa con el resto de ítems y presentaba, a su vez, una correlación baja con la totalidad de la dimensión (El proyecto incluye entre sus objetivos la publicación bilingüe o multilingüe de material informativo).

- De la última dimensión propuesta interculturalidad como valor positivo, tres ítems presentaron correlaciones no significativas con muchos de los ítems restantes.

\subsection{Fiabilidad}

El análisis de fiabilidad de cada una de las dimensiones y del cuestionario global se ha realizado mediante el análisis de la consistencia interna, ya que se consideró el procedimiento más adecuado y ajustado a las características de la temática y de los objetivos establecidos. El coeficiente Alfa sería un estimador directo del coeficiente de fiabilidad del test, sin embargo, en la mayoría de las situaciones de medida esta suposición es insostenible, por lo que la afirmación se restringe y se dice que alfa es el límite inferior del coeficiente de fiabilidad (Carmona, Lozano, Pérez y Sánchez, 2006).

Los valores obtenidos en cada una de las dimensiones en un primer momento, previo a la depuración del instrumento (sin la eliminación de ningún ítem) y, posteriormente, con el instrumento definitivo se pueden contemplar en la Tabla 3. La fiabilidad del instrumento final es bastante elevada, alcanzando el valor de 0.92 y la fiabilidad de cada una de las dimensiones es bastante aceptable, superando en todos los casos el valor de 0,7 recomendado (Nunnaly y Bernstein, 1995).

\subsection{Evidencias de validez}

Se procedió posteriormente al análisis de evidencias de validez basadas en la estructura interna del test, para lo que se realizaron análisis factoriales exploratorios, tomando el criterio de Kaisser y con rotación oblimin. El objetivo del análisis factorial exploratorio es la «determinación del mínimo número de estructuras necesarias para explicar la máxima varianza observada» (Elosua, 2003). La posición de partida era la consideración de seis dimensiones: teórica, socio-política, ético, ideológica,

\begin{tabular}{|l|c|c|}
\hline \multicolumn{1}{|c|}{ Dimensión } & $\begin{array}{c}\text { Alfa } \\
\text { anterior }\end{array}$ & $\begin{array}{c}\text { Alfa } \\
\text { actual }\end{array}$ \\
\hline Dimensión teórica & 0,88 & 0,88 \\
Factor contextual & 0,79 & 0,79 \\
Factor Concepción diversidad & 0,83 & \\
Factor Elementos de diver- & & 0,85 \\
sidad & 0,88 & 0,89 \\
Dimensión ético-ideológica & 0,75 & 0,83 \\
Dimensión socio-política & 0,73 & 0,84 \\
Dimensión empatía-intercul- & & \\
tural & 0,73 & 0,86 \\
\hline
\end{tabular}

Tabla 3. 
empatía intercultural e interculturalidad como valor positivo. Se realizaron los análisis factoriales exploratorios de cada una de las dimensiones y de la escala global, contando, en todas las dimensiones, con la adecuación de la prueba de Kaisser (KMO) y la esfericidad de Bartlett para realizar estos análisis.

En cuando a la dimensión teórica, las pruebas KMO fueron de 0,6 para el factor contextual, 0,76 para el de concepción de la diversidad y de 0,77 en el de elementos de diversidad. Las pruebas de esfericidad de Bartlett fueron estadísticamente significativas en los tres factores $(p=0,000)$.

El análisis factorial exploratorio del aspecto contextual extrajo un único factor que explica un 61,3 por ciento de la varianza; en el aspecto de concepción de la diversidad se obtuvo también un único factor que explicaba un 59,32 por ciento de la varianza pero, debido a la baja saturación de un ítem (Se consideran las diferencias culturales de los agentes implicados en el proyecto/actividad, actores y beneficiarios) y apoyándonos en su mal funcionamiento en las correlaciones y el aumento de fiabilidad que se producía con su eliminación, se procedió a suprimirlo, aumentando con ello la varianza total explicada por el factor a 71,49 por ciento. Este ítem se suprimió porque, aunque el contenido puede resultar de gran relevancia, existen otros ítems que dan respuestas concretas a este argumento, lo que dificulta los buenos resultados psicométricos y puede ser repetitivo para las personas que cumplimentan la escala. El último componente de la dimensión teórica, elementos de diversidad, presentaba inicialmente dos factores que explicaban el 55,36 por ciento y el 14,12 por ciento de la varianza respectivamente. Existían dos ítems (el primero, Se consideran los idiomas hablados/escritos de los agentes implicados en el proyecto/actividad y el segundo, Se considera la apariencia física de los agentes implicados en el proyectos/actividad) que, además de ser los que presentaban peores correlaciones, saturaban en otro factor, por lo que decidimos la eliminación de ambos aumentando de este modo la varianza explicada a un 66,1 por ciento y con la extracción de un único factor.

En la dimensión ético-ideológica, teniendo en $\mathrm{KMO}(0,60)$ y Bartlett (significativa $\mathrm{p}=0,00)$ se realizó el análisis factorial explora- torio, obteniéndose en primer lugar cuatro factores. De manera progresiva, se procedió a la eliminación de ítems con saturaciones en más de un factor (encontrándose entre ellos los cinco ítems que presentaron inicialmente bajas correlaciones). Finalmente se obtuvo una extracción unifactorial que explica el 53,9 por ciento de la varianza.

La dimensión socio-politica (determinante 0,33 ), a la que según las pruebas de Bartlett (significativa valor $\mathrm{p}=0,00)$ y de $\mathrm{KMO}(0,75)$ era apropiado realizar también un análisis factorial exploratorio, mostró en un primer análisis tres factores que explicaban el 44,51 por ciento, el 16,04 por ciento y 13,30 por ciento de la varianza cada uno de ellos. Teniendo en cuenta las correlaciones, el valor de alfa si se elimina algún elemento y la elevada saturación en otro factor, se procedió a la eliminación de un ítem, que además se corresponde con uno de los dos que no presentaban correlaciones significativas con el resto de ítems de su dimensión (El proyecto/actividad facilita información a las minorias culturales exclusivamente en el idioma de la comunidad de acogida). Con ello, obtuvimos dos factores, de los cuales, el segundo de ellos estaba formado únicamente por un ítem (El proyecto/actividad facilita información a los destinatarios exclusivamente en su propio idioma) que fue eliminado no sólo por su ubicación factorial, sino también por su contenido semántico y por sus bajas correlaciones. La solución final de esta dimensión, con la supresión de estos dos ítems, aporta un único factor que logra explicar el 57,53 por ciento de la varianza.

En la dimensión empatía intercultural (determinante es 0,58 ) con un valor para $\mathrm{KMO}$ de 0,71 y siendo significativa la prueba de Bartlett, el primer análisis factorial exploratorio ofreció una solución con dos factores (explicaban de 59,82 por ciento y 20,70 por ciento de la varianza) donde únicamente un ítem saturaba en un factor distinto al que lo hacía el resto (El proyecto incluye entre sus objetivos la publicación bilingüe o multilingüe de material informativo). Una vez que se eliminó este ítem, la varianza explicada por un único factor aumentó hasta el 74,21 por ciento. Una decisión relevante en el proceso de investigación, fue la eliminación de la dimensión «interculturalidad como valor positivo», ya que la estructura uni- 
factorial se mantenía únicamente con la eliminación de muchos ítems. Basándonos en el contenido semántico optamos por la reubicación de dos de los ítems en otras dimensiones que también consideramos afines en cuanto a contenido. Uno de los ítems (El proyecto/ actividad condena explícitamente estereotipos $y$ mensajes racistas) fue incluido en concepción de la diversidad de la dimensión teórica, que seguía manteniendo, con esta inclusión, la estructura unifactorial. El otro ítem (Se consideran los idiomas hablados/escritos de los agentes implicados en el proyecto/actividad) fue finalmente ubicado en el aspecto contextual de la dimensión teórica, debido a la mayor proximidad conceptual con esta dimensión y que también mantuvo una solución unifactorial con la incorporación de este ítem.

Los resultados se pueden contemplar en las Tablas 4 a 8, considerando que para la eliminación de los ítems se tuvieron en cuenta las correlaciones no significativas, la mala saturación (tanto en valor como en ubicación de factor) y el contenido semántico. Este proceso de eliminación modificó la varianza final explicada en cada dimensión, tal y como muestra la Tabla 10.
El instrumento construido (Escala BPI) para la medición de proyectos que quieran evaluarse como buenas prácticas interculturales presenta adecuadas propiedades psicométricas, con evidencias de validez de constructo y elevada fiabilidad basada en la estructura interna de la escala.

\section{Discusión}

En el marco de las dinámicas sociales contemporáneas y la actual dinámica en la composición de nuestras poblaciones, considerar la dimensión cultural en las intervenciones sociales (en todos sus niveles, ámbitos y dimensiones), es uno de los principales retos existentes para conseguir intervenciones exitosas y luchar contra prácticas segregacionistas, asimilacionistas e incluso racistas/xenófobas.

La interculturalidad es un concepto que se encuentra en plena vigencia y que está experimentando un crecimiento en relación a su estudio y conceptualización, pero que carece de instrumentos de medida científicos y validados empíricamente que avalen y apoyen las decisiones teóricas y de intervención, dando una nueva visión y respaldo a las acciones que se implementan.

\begin{tabular}{|l|c|c|c|c|c|}
\hline $\begin{array}{c}\text { Contextual } \\
\text { AFE 1 }\end{array}$ & Componente & $\begin{array}{c}\text { Concepción } \\
\text { diversidad } \\
\text { AFE 1 }\end{array}$ & Componente & $\begin{array}{c}\text { Concepción } \\
\text { diversidad } \\
\text { AFE 2 }\end{array}$ & Componente \\
\hline D. teorica 1 &, 799 & D. teórica 8 &, 885 & D. teórica 8 &, 901 \\
D. teorica 5 &, 792 & D. teórica 7 &, 870 & D. teórica 7 &, 860 \\
D. teórica 17 &, 759 & D. teórica 4 &, 836 & D. teórica 4 &, 847 \\
D. eticoide13 &, 706 & D. teórica 6 &, 758 & D. teórica 6 &, 768 \\
D. teórica 3 &, 680 & D. teórica 2 &, 390 & & \\
\hline
\end{tabular}

Tabla 4. Factor contextual y concepción de diversidad de la dimensión teórica.

\begin{tabular}{|l|c|c|c|c|}
\hline \multirow{2}{*}{ AFE 1 } & \multicolumn{2}{|c|}{ Componente } & \multirow{2}{*}{ AFE 3 } & Componente \\
\cline { 2 - 4 } & $\mathbf{1}$ & $\mathbf{2}$ & D. teórica 9 &, 876 \\
\hline D. teórica 12 &, 890 & & D. teórica 12 &, 873 \\
D. teórica 10 &, 849 & & D. teórica 10 &, 866 \\
D. teórica 9 &, 849 & & D. teórica 11 &, 795 \\
D. teórica 11 &, 767 & & D. teórica 14 &, 742 \\
D. teórica 14 &, 747 & & D. teórica 16 &, 710 \\
D. teórica 16 &, 713 &,- 514 & & \\
D. teórica 13 & &, 730 & & \\
D. teórica 15 & & & & \\
\hline
\end{tabular}

Tabla 5. Factor elementos de diversidad de la dimensión teórica. 


\begin{tabular}{|c|c|c|c|c|c|c|}
\hline \multirow{2}{*}{ AFE 1} & \multicolumn{4}{|c|}{ Componente } & \multirow{2}{*}{ AFE 9} & \multirow{2}{*}{ Componente } \\
\hline & 1 & 2 & 3 & 4 & & \\
\hline D. eticoide 7 &, 800 & ,410 & & & D. eticoide 10 &, 871 \\
\hline D. eticoide 10 & ,784 & & & & D. eticoide 7 & ,854 \\
\hline D. eticoide 5 & ,739 & &,- 508 & & D. eticoide 3 & ,812 \\
\hline D. eticoide 3 & 670 & & & & D. eticoide 5 &, 769 \\
\hline D. eticoide 2 &,- 667 & & & & D. eticoide 9 &, 562 \\
\hline D. eticoide 9 & ,653 & & &,- 495 & D. eticoide 11 & ,425 \\
\hline D. eticoide 13 & 648 & & & & & \\
\hline D. eticoide 8 & ,612 & &, 595 & & & \\
\hline D. eticoide 11 & ,549 & & & & & \\
\hline D. eticoide 4 & ,522 &,- 509 & & & & \\
\hline D. eticoide 6 & ,497 - - & ,491 &,- 496 & & & \\
\hline D. eticoide 1 & & ,695 & & & & \\
\hline D. eticoide 12 & ,426 &,- 497 & & ,531 & & \\
\hline
\end{tabular}

Tabla 6. Dimensión ético-ideológica.

\begin{tabular}{|l|c|c|c|c|c|}
\hline \multirow{2}{*}{ AFE 1 } & \multicolumn{3}{|c|}{ Componente } & \multirow{2}{*}{ AFE 3 } & Componente \\
\cline { 2 - 3 } & $\mathbf{1}$ & $\mathbf{2}$ & $\mathbf{3}$ & & \\
\hline D. sociopol 2 &, 922 & & & D. sociopol 2 &, 903 \\
D. sociopol 7 &, 809 & & & D. sociopol 7 &, 790 \\
D. sociopol 1 &, 804 & & & D. sociopol 5 &, 767 \\
D. sociopol 6 &, 640 & & & D. sociopol 1 &, 766 \\
D. sociopol 5 &, 635 &, 506 & & D. sociopol 8 &, 663 \\
D. sociopol 3 &, 527 &, 910 & & D. sociopol 6 &, 631 \\
D. sociopol 8 & & \multirow{2}{*}{, 972} & & \\
D. sociopol 4 & & & & \\
\hline
\end{tabular}

Tabla 7. Dimensión socio-política.

\begin{tabular}{|c|c|c|c|c|}
\hline \multirow{2}{*}{ AFE 1 } & \multicolumn{2}{|c|}{ Componente } & \multirow{2}{*}{ AFE 2 } & $\mathbf{1}$ \\
\cline { 2 - 3 } & $\mathbf{1}$ & $\mathbf{2}$ & &, 928 \\
\hline EmpatiaInterc. 2 &, 916 & & EmpatiaInterc. 2 &, 876 \\
EmpatiaInterc. 5 &, 864 & & EmpatiaInterc. 5 &, 828 \\
EmpatiaInterc. 1 &, 831 & & EmpatiaInterc. 4 &, 809 \\
EmpatiaInterc. 4 &, 821 & & EmpatiaInterc. 1 & \\
EmpatiaInterc. 3 & & \multirow{2}{*}{, 978} & & \\
\hline
\end{tabular}

Tabla 8. Dimensión empatía intercultural.

El sentido de este proyecto en el que se ubica la construcción y validación de este instrumento se relaciona con la preocupación por responder de manera eficaz a la realidad que se localiza en un espacio de diversidad en el que intervienen actores de sectores y naturaleza diferentes, tanto de las administraciones públi- cas, que o bien subvencionan la realización de acciones o bien las ejecutan directamente, como de la iniciativa social que desarrollan gran parte de los proyectos y acciones centrados en la gestión de la diversidad.

La Escala BPI que se presenta es un instrumento novedoso, dada la inexistencia de nin- 


\begin{tabular}{|c|c|c|c|}
\hline \multirow{2}{*}{ AFE 1 } & \multicolumn{3}{|c|}{ Componente } \\
\cline { 2 - 4 } & $\mathbf{1}$ & $\mathbf{2}$ & $\mathbf{3}$ \\
\hline Interc.val.positivo 3 &, 862 & & \\
Interc.val.positivo 7 &, 793 & & \\
Interc.val.positivo 1 &, 717 & &, 475 \\
Interc.val.positivo 4 & &, 857 & \\
Interc.val.positivo 6 & &, 791 & \\
Interc.val.positivo 5 & &, 737 &, 534 \\
Interc.val.positivo 2 & & &, 902 \\
\hline
\end{tabular}

Tabla 9. Dimensión interculturalidad como valor positivo.
A pesar de estas múltiples ventajas y potencialidades, no hay que obviar la existencia de ciertas limitaciones que deben ser trabajadas en el futuro y en las que el equipo investigador tiene intención de profundizar. En este sentido se debe mejorar el análisis psicométrico avanzando hacia comprobación de la estructura de la escala con análisis factoriales confirmatorios. Igualmente hay que avanzar en el conocimiento de evidencias de validez del instrumento empleado, más allá de la validez de contenido mostrada. Hay que continuar profundizando en la búsqueda bibliográfica de investigaciones si-

\begin{tabular}{|c|c|c|c|c|c|c|}
\hline Dimensión & $\begin{array}{l}\mathrm{N}^{\circ} \\
\text { ítems } \\
\text { inicial }\end{array}$ & $\begin{array}{c}\mathrm{N}^{\circ} \\
\text { factores }\end{array}$ & $\begin{array}{l}\text { Varianza } \\
\text { explicada }\end{array}$ & $\begin{array}{l}\mathrm{N}^{\mathbf{0}} \\
\text { ítems } \\
\text { final }\end{array}$ & $\begin{array}{l}\text { Varianza } \\
\text { explicada }\end{array}$ & $\begin{array}{c}\text { Ítems } \\
\text { cuestionario } \\
\text { final }\end{array}$ \\
\hline Teórica F. Contextual & 4 & 1 & $61,3 \%$ & 5 & $61,3 \%$ & $1-5$ \\
\hline Teórica F. Concepción diversidad & 5 & 1 & $59,32 \%$ & 5 & $71,49 \%$ & $6-10$ \\
\hline Teórica F. Elementos diversidad & 8 & 2 & 55,36 y $14,12 \%$ & & $66,1 \%$ & $11-16$ \\
\hline Ético-ideológica & 13 & 4 & & 5 & $53,9 \%$ & $17-21$ \\
\hline Socio-política & 8 & 3 & $\begin{array}{c}44,51 \%, 16,04 \% \\
\text { y } 13,30 \%\end{array}$ & 7 & $57,53 \%$ & $22-28$ \\
\hline Empatía intercultural & 5 & 2 & 59,82 y $20,70 \%$ & 4 & $74,21 \%$ & $29-32$ \\
\hline $\begin{array}{l}\text { Interculturalidad como valor po- } \\
\text { sitivo }\end{array}$ & 7 & 3 & & Eliminada & & \\
\hline
\end{tabular}

Tabla 10.

gún otro que permita medir a nivel de actuación e intervención estas características de prácticas interculturales.

La disponibilidad de este instrumento puede abrir la puerta a nuevas investigaciones en otros ámbitos geográficos, analizando las relaciones entre dimensiones con múltiples variables tanto de los proyectos como externas a los mismos, procesos que además servirán para seguir comprobando aspectos métricos de la escala y su aplicación práctica. También puede resultar de utilidad de cara a los procesos evaluativos de las entidades que estén interesadas en conocer los resultados de las acciones que se llevan a cabo en diferentes sectores de actuación relacionados con la interculturalidad. milares que permitan conectar variables en modelos estructurales y con ello dar respuestas más integrales a las necesidades de evaluación de la interculturalidad en las pautas de intervención social. Igualmente es posible mejorar el proceso de aplicación para valorar métodos que permitan reducir la subjetividad de la persona responsable de cumplimentar la escala en relación a las pautas objetivas de los proyectos que son objeto de evaluación.

Las líneas de futuro del equipo investigador se dirigen a la profundización de aspectos psicométricos de la escala, analizando otras evidencias de validez (concurrente, discriminante, de relación con otras variables) y seguir avanzando, como se puede deducir, en la aplicación social del instrumento. ${ }^{1}$

${ }^{1}$ Los autores del texto quieren expresar su agradecimiento a la Dirección General de Políticas Migratorias de la Consejería de Gobernación de la Junta de Andalucía por haber financiado en el año 2006 el proyecto de investigación «Mapa de las buenas prácticas interculturales en Andalucía», de donde se han extraído los datos comunicados en este trabajo, así como a todos los proyectos andaluces que han colaborado con el mismo. 


\section{Referencias bibliográficas}

APA (2003). Guidelines on multicultural education, training, research, practice and organizational change for psychologists. American Psychologists, 58 (5), 377-402.

Barret, M. (2003). Guidelines for intercultural best practice in local service provision. Published by the Intercultural Working Group of the North West Inner City, pp.13 y ss. Recuperado de http://www.accessireland.ie/Guidelines.pdf. (25/3/2011).

Bernal, G. y Sáez-Santiago, E. (2006). Culturally centered psychosocial interventions. Journal of Community Psychology, 34 (2), 121-132.

Bennet, M. J. (1986). A developmental approach to training for intercultural sensitivity. International Journal of Intercultural Relations 10, 179- 196.

Cabrera, P. J. (Mayo, 2003). La importancia de las Buenas Prácticas en los proyectos sociales. Trabajo presentado en el Seminario 'Buenas Prácticas en la Inclusión Social'. Cruz Roja Española. Madrid.

Carmona, J., Lozano, O. M., Pérez, P. J. y Sánchez, M. (2006). Análisis de Ítems y Validación de Pruebas Psicológicas y Educativas con SPSS y Amos. Huelva: Universidad de Huelva.

Chen, G. M. y Starosta, W. J. (Noviembre, 2000). The Development and Validation of the Intercultural Sensitivity Scale. Trabajo presentado en The Annual Meeting of the National Communication. Seattle, WA. Recuperado de: http://eric.ed.gov/ERICWebPortal/Home.portal;jsessionid=GbNH1LHMpQQQX1MTPxcKLzQQg921KkWchrbGV0b0VTT41JrzvVN8!779436061? _nfpb=trae\&_pageLabel=ERICSearchResult\&_urlType=action\&newSearch=true\&ERICExtSearch_SearchType_0=au\&ERICExtSearch_SearchValue_0=\%22Chen+Guo-Ming\%22. $(16 / 12 / 2006)$.

Elosua, P. (2003). Sobre la validez de los tests. Psicothema 15 (2), 315-321.

FEAPS (2003). Buenas prácticas FEAPS. ¿Qué son y cómo se valoran? Red de Calidad. Recuperado de: http://www.feapscv.org/web/pdf/que_es_buena_practica.pdf. (10/12/2007).

Fritz, W., Mollenberg, A. Yy Chen, Guo-Ming (Julio,2001). Measuring intercultural sensitivity indifferent cultural context. Trabajo presentado en Biannual Meeting of the International Association for Intercultural Communication Studies. Hong Kong. Recuperado de http://eric.ed.gov/ERICWebPortal/Home.portal;jsessionid=GbNH1LHMpQQQXIMTPxcKLz QQg921KkWchrbGV0b0VTT41JrzvVN8!779436061?_nfpb=true\&_pageLabel=ERICSearchResult\&_urlType=action\&newSearch=true\&ERICExtSearch_SearchType_0=au\&ERICExtSearch_SearchValue_0=\%22Chen+Guo-Ming\%22. (15/7/2007).

Jordán, J.A. (1996). Propuestas de Educación Intercultural para Profesores. Barcelona: Ed. CEAC.

Julve, M. y Palomo, B. (2005). La competencia comunicativa intercultural en la prestación de servicios. Glosas didácticas, 15, 26-38.

Liévano, B. M. (2003). Intervenciones para la adquisición de competencias interculturales en Repetto, E. (coord.), Modelos de Orientación e Intervención Psicopedagógica (424-452), 2. Madrid: UNED.

Martínez, M.F., Martínez, J. y Calzado V.(2006). La Competencia Cultural como referente de la Diversidad Humana en la Prestación de Servicios y la Intervención Social. Intervención Psicosocial, 15(3), 331-350.

Martuccelli, D. (2004). Representaciones e interculturalidad. Lo intercultural ante la prueba de la dinámica entre exclusión e integración social. Revista Cidob D’afers Internacionals, 66-67, 53-68.

Maya, I. (2002). Estrategias de entrenamiento de las habilidades de comunicación intercultural. Portularia: Revista de Trabajo Social, 2, 91-108.

Nunnally, J.C. y Bernstein, I.J. (1995). Análisis Factorial Exploratorio II: Rotación y otros temas. En Nunnally, J.C. y Bernstein, I.J., Teoría de la Psicometría.Nueva York: MacGraw Hill.

Panikkar, R. (2006). Decálogo: cultura e interculturalidad. Cuadernos Interculturales, 6, 129-130.

Pratt, H.D y Apple R.W. (2007). Cross-cultural assessment and management in primary care. Primary care: clinics in office practice, 34 (2), 227-242. 
Pedersen, P.D. (2003). Cross-cultural counseling: Developing culture-centered interactions. En: G. Bernal, J.E. Trimble, A.K. Burlew y F.T.L. Leong (eds.), Handbook of racial and ethnic minority psychology (pp. 487-503). Thousand Oaks, CA: Sage.

Rogers-Sirin, L. (2008). Approaches to multicultural training for professionals: a guide for choosing an appropriate program. Profesional Psychology: research and practice, 39(3), 313-319.

Trickett, E. J., Watts, R. J., y Birman, D. (1994). Toward an overarching framework for diversity. En: E. J. Trickett, Watts, R. J.,y y Birman, D. (eds.), Human diversity: Perspectives and people in context (pp. 7-26). San Francisco: Jossey-Bass.

U.S. Department of Health and Human Service (2001). National Standars for Culturally and Linguistically Appropriate Services in Health Care. Washington D.C.: Office of Minority Health.

Vázquez, O. (2001). Acción intercultural y trabajo social. Cuadernos de Trabajo Social, 14, 29-43.

Vázquez, O. (2005). La competencia intercultural y las habilidades de intervención en mediación social intercultural. Manual de Atención social al inmigrante (pp.107-125). Córdoba: Almuzara.

Vázquez, O., Fernández, M. Fernández, M. Vaz, P. y León, J.C. (2008). Políticas públicas, calidad de vida e interculturalidad. Revista del Ministerio de Trabajo e Inmigración, 75, 201-211.

Whealin, J.M. y Ruzek, J. (2008). Program evaluation for organizational cultural competence in mental health practice. Professional Psychology: research and practice, 39(3), 320-328. 